\title{
Drug Seeking Behavior of Amphetamine Addicted Sprague-Dawley Rats Is Eliminated after Nutritional Supplementation
}

\author{
Annice Webber-Waugh', Karen Thaxter Nesbeth', Pauline Anderson-Johnson'2, \\ Ajibike Salako-Akande ${ }^{3}$, Helen Asemota ${ }^{1,4}$, Lauriann Young ${ }^{*}$ \\ ${ }^{1}$ Department of Basic Medical Sciences, The University of the West Indies (UWI), Mona Campus, Kingston, Jamaica \\ ${ }^{2}$ UWI School of Nursing, Mona Campus, Kingston, Jamaica \\ ${ }^{3}$ Getwele Natureceuticals LLC, Halethorpe, MD, USA \\ ${ }^{4}$ The Biotechnology Centre, UWI, Kingston, Jamaica \\ Email: *lauriann.young@uwimona.edu.jm
}

How to cite this paper: Webber-Waugh, A., Thaxter Nesbeth, K., Anderson-Johnson, P., Salako-Akande, A., Asemota, H. and Young, L. (2017) Drug Seeking Behavior of Amphetamine Addicted Sprague-Dawley Rats Is Eliminated after Nutritional Supplementation. Journal of Behavioral and Brain Science, 7, 585-597.

https://doi.org/10.4236/jbbs.2017.712041

Received: July 21, 2017

Accepted: November 19, 2017

Published: November 22, 2017

Copyright (c) 2017 by authors and Scientific Research Publishing Inc. This work is licensed under the Creative Commons Attribution International License (CC BY 4.0).

http://creativecommons.org/licenses/by/4.0/

\begin{abstract}
Aim: The effectiveness of nutritional supplementation on drug-seeking behavior of amphetamine-addicted rats during withdrawal was investigated using a biased conditioned place preference (CPP) paradigm. Method: Twenty-four male Sprague-Dawley rats exhibiting baseline preference for the black chamber during a 20-minute pre-conditioning exploration of the CPP box completed the study. On alternate days of an 8-day schedule, twelve rats (Group AMP) were randomly selected, given either amphetamine sulfate $(5 \mathrm{mg} / \mathrm{ml}$, i.p.) and confined to the white chamber; or vehicle ( $1 \mathrm{ml}$ saline, i.p.) and confined to the black chamber. A significant increased percentage time spent and number of entries made by Group AMP to the drug-paired, white chamber on the test day confirmed amphetamine addiction. Group AMP subsequently received increasing doses of amphetamine over 6 days. Following acute drug withdrawal, their CPP performance was compared with that of vehicle treated rats (Group SAL). Groups AMP and SAL were equally divided and randomly assigned to animals fed chow reconstituted with the nutritional supplement (AMP-S and SAL-S) over 8 weeks or standard rat chow (AMP-N and SAL-N). CPP performances for all rats were determined blindly from video recordings following this period. Results: Nutritionally supplemented, amphetamine withdrawn rats (AMP-S) exhibited significantly decreased percentage entries and time spent in the white chamber $(\mathrm{p}<0.05)$, indicating preference for the black chamber and diminished drug-seeking behavior. ANOVA revealed that after 8 weeks of dietary supplementation, AMP-S rats behaved like drug-naïve, control animals. Conclusion: Drug-seeking behavior by amphetamine-addic-
\end{abstract}


ted animals was eliminated after treatment with a nutritionally supplemented diet.

\section{Keywords}

Amphetamine, Conditioned Place Preference, Nutritional Supplement, Addiction, Animal Model

\section{Introduction}

Substance dependence and substance abuse, previously considered to be separate entities, have recently been incorporated into the disorder classified in the DSM-5 as Drug Addiction [1] [2]. Drug addiction, which is a recognized brain disease, is a chronically relapsing disorder, characterized by compulsion to seek and take the drug, loss of control in limiting intake, and emergence of a negative emotional state that includes dysphoria, anxiety, and irritability when access to the drug is prevented. There are currently three recognized stages of drug addiction, namely: 1) the basal ganglia-driven binge/intoxication stage; 2) an extended amygdala-driven withdrawal/negative affect stage; and 3) a prefrontal cortex-driven preoccupation/anticipation stage. Each stage is associated with disturbances in a specific series of neural subsystems, and involves complex neurotransmitter interactions [1].

The ability of a drug of abuse to induce reward or intoxication is initially, although not solely, related to its ability to increase levels of dopamine (DA), particularly in the nucleus accumbens of the basal forebrain. This site-specific increase in DA then enables the individual to predict reward, imprint incentive value to reinforcers and rapidly learn reward associations [3]. This means that the power to create an addiction does not merely lie in induction of timely hedonic pleasure, but in the evolution of complex neuroadaptations, involving DA and other neurotransmitters and receptors such as glutamate, GABA, endocannabinoids, and opioids. They synergistically produce the behaviors associated with addiction [1]. Research on addiction trajectories has shown that the continued use of an abused substance gradually impairs neuronal function, eventually impacting the very capacity of the drug dependent person or addict to exert free will [2]

Amphetamine is a powerful stimulant of the central nervous system (CNS) with high addictive potential. It is also highly anorexigenic. The amphetamine-induced peptides, known as cocaine and amphetamine-regulated transcript (CART) 1 and 2, have been identified as being specifically produced upon administration of amphetamine to rodents. They decrease food intake and increase energy expenditure [3]. CART is widely expressed in the CNS, including brain regions controlling food intake, and direct administration of CART decreases food intake [4]. CART is believed to increase metabolism by causing an increase in the release of thyrotropin-releasing hormone (TRH) that in turn 
stimulates release of thyroid stimulating hormone to cause an increase in heat production by muscle and adipose tissue [5].

Amphetamine abusers therefore experience reduced food intake, increased caloric expenditure, and suffer from a malnutrition related to starvation. Starvation itself induces protein and fat catabolism that leads to loss of organ volume and function. Food restriction causing weight loss below approximately 15 to 20 percent of ideal body weight increases the potential for development of gastroparesis [6], with the associated nausea, early satiety and anorexia which may add to the nutritional insult of amphetamine abuse [7]. Furthermore, persons with gastroparesis are at high risk of developing small bowel bacterial overgrowth and the consequent increased gut transit, maldigestion and malabsorption; painting a picture of an addict caught in a cycle of inadequate nutrition [6].

The gastrointestinal, neuronal and caloric perturbations resulting from amphetamine abuse are indicators that nutrition therapy is vital for individuals with substance use disorders. Furthermore, disordered and dysfunctional eating patterns pervade attempts at abstinence, potentially worsening the withdrawal experience of the addict. Centers and caregivers are more likely to have improved results if nutrition and abstinence are addressed simultaneously. Thus, specific macro- and micro-nutrient supplementation is recommended early in the treatment cycle to supplement calories, neurotransmitter regulation and restoration of gut health [8].

This study therefore investigated the effectiveness of a proprietary formulation of a nutritional supplement on drug-seeking behavior in drug-withdrawn rodents using an amphetamine addicted rat model, as confirmed by the conditioned place preference (CPP) paradigm, an accepted gold standard for rodent addiction studies.

\section{Materials and Methods}

A biased CPP paradigm was used to create an amphetamine rat addiction model to test the drug-seeking behavioral response to nutritional supplementation during drug withdrawal. A three (3) chambered CPP apparatus, measuring 75 $\mathrm{cm} \times 30 \mathrm{~cm} \times 25 \mathrm{~cm}$, was employed. The single black, white and grey chambers could each be isolated from the other by a sliding door on either side of the central grey chamber. The box had a Perspex lid to facilitate viewing and video recording. The floor of the white chamber was covered with shiny transparent Perspex; while the floor of the black chamber was covered with wire mesh and saw-dust, creating two contrasting chambers with distinct visual and tactile cues, representing a biased paradigm. Figure 1 shows a diagram of the CPP box used in this study. The apparatus was thoroughly cleaned with a dilute solution of disinfectant upon completion of exploration by each rat.

\subsection{Subjects}

Twenty four (24) three (3) month old male Sprague-Dawley rats weighing approximately 250 - $350 \mathrm{~g}$, were obtained following institutional ethical approval of 


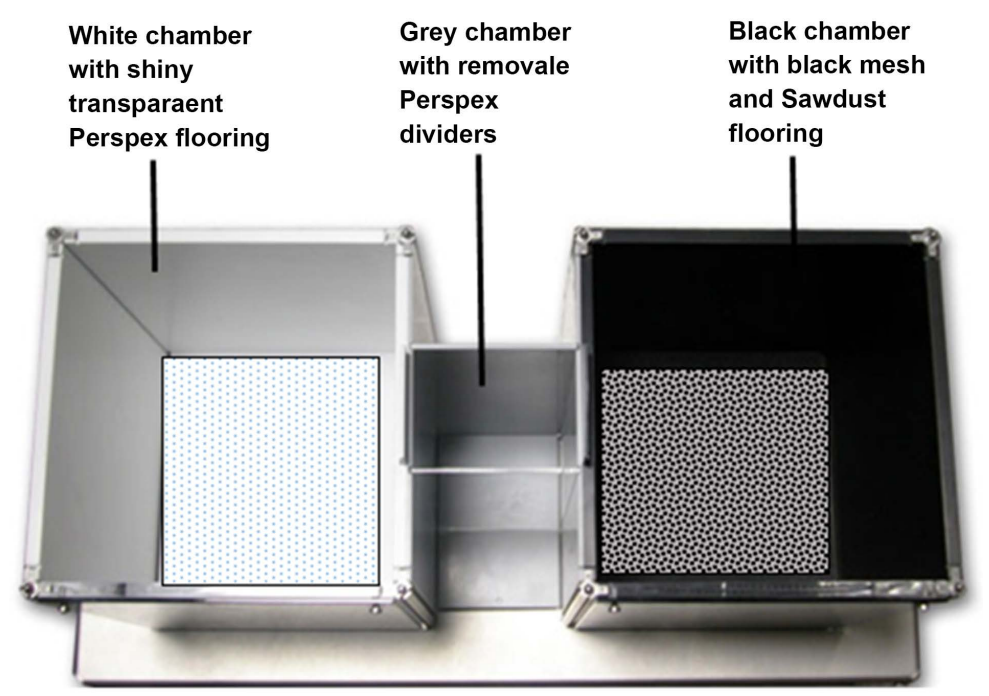

Figure 1. Diagram of the conditioned place preference apparatus employed for this study.

the protocol (UHWI/UWI/FMS Ethics Committee, AN02/2007/2008). Animals were housed in pairs, in a normal 12:12 hour light: dark cycle and fed food and water ad libitum.

\subsection{The Conditioned Place Preference Paradigm}

\section{Phase 1: Pre-conditioning, Selection and Group Assignments}

Animals selected for inclusion in the experiment were allowed an initial twenty (20) minute exploration of the CPP apparatus, and scores of chamber entries and time spent in each chamber were recorded manually and correlated with the video recorded data. An entry was recorded when all four paws of the rat were placed in a chamber. Animals displaying at least $80 \%$ preference for the black CPP chamber during pre-conditioning, assessed as greater number of entries and time spent in the black chamber, were selected and randomly assigned to one of two treatment groups, AMP or SAL, each containing twelve (12) rats [9] [10].

\section{Phase 2: Conditioning}

During the conditioning phase, rats assigned to the amphetamine treatment group (Group AMP) received intra-peritoneal injections (i.p.) of $5 \mathrm{mg} / \mathrm{kg} / \mathrm{ml}$ of amphetamine sulfate (Sigma, USA) dissolved in physiological saline (0.9\%). This dose has been shown to produce place preference in a number of studies [9] [10]. Rats in Group SAL were given only vehicle ( $1 \mathrm{ml} 0.9 \%$ saline, i.p.).

Conditioning consisted of 8 days of daily, group-assigned i.p. injections, followed immediately by individual confinement to the white, "non-preferred" or black "preferred" chamber of the CPP box for thirty (30) minutes. On days 1, 3, 5 and 7, Group AMP received amphetamine sulfate (Sigma, USA) while nondrug exposed, vehicle treated rats received saline (Group SAL). Each rat in Groups AMP and SAL was then confined individually to the white "non-pre- 
ferred" chamber. On the four alternate days (i.e., Days 2, 4, 6, 8), all animals received an injection of saline ( $1 \mathrm{ml}$, i.p.) and were confined individually for 30 minutes in the black "preferred" chamber. Thus, Group AMP rats were conditioned to pair amphetamine with the white chamber and saline with the black chamber [9] [10]. On Day 9, rats were placed individually in the grey chamber, sliding doors then removed, and allowed to explore the CPP box. The number of entries and time spent in the white chamber were recorded over 20 minutes and the percentage entries and time spent calculated from the total. Data obtained revealed that all animals assigned to Group AMP exhibited a significant and positive CPP drug-seeking behavior for the white chamber (Figure 2).

Phase 3: Amphetamine Addiction and Drug Withdrawal

The addiction phase then ensued, with daily doses of amphetamine sulfate (5, $5,6,6,7,7 \mathrm{mg} / \mathrm{kg} / \mathrm{ml}$, i.p.) that increased in concentration over a 6 -day period. Group SAL rats received only vehicle (i.p.) over the same period and represented the non-drug exposed controls for the addiction phase of the study.

Rats were subsequently taken through a period of three days without drug, vehicle or CPP exposure, and were fed standard rat chow with water ad libitum. On day 4, following drug withdrawal, each animal was individually placed in the grey start chamber and allowed a 20-minute period of exploration of the CPP box after the sliding doors were removed (Figure 2). The number of entries made into, and the time spent in each chamber, were recorded manually for all rats and the data compared with scores obtained from video recordings of exploration of the CPP box.

Phase 4: Oral Nutritional Supplementation

The orally administered, nutritional supplementation regimen used in this study consisted of three separate pharmaceutical grade components: 1) a mega vitamin with higher levels of calcium and magnesium;2) omega 3 and 6 fish oils; and 3) a supplement capsule consisting of basic amino acids with higher levels of tyrosine (Manufacturer: All The Whey, Inc. Paoli PA, USA).

Nutritionally supplemented animals were proportionally fed a combination of the named component, at $0.123 \mathrm{~g} /$ day, $0.123 \mathrm{~g} /$ day and $0.0408 \mathrm{~g} /$ day, respectively, over a period of 8 weeks. These doses were calculated based on a $350 \mathrm{~g}$ rat, after proportions previously used in other studies for administration to a $75 \mathrm{~kg}$ man were scaled down [11].

Preparation of the nutritional supplement for oral treatment involved crushing and weighing the megavitamins; whereas, the fish oil tablets were opened and drained into a calibrated beaker and the relevant volumes removed. Supplement capsules were also opened and the powder weighed for correct dosing. All animals were individually caged for the duration of the supplementation period.

The initial Groups AMP and SAL were further equally divided, assigned a numerical code and randomly assigned to either the supplemented diet or standard rat chow group, as indicated below: 


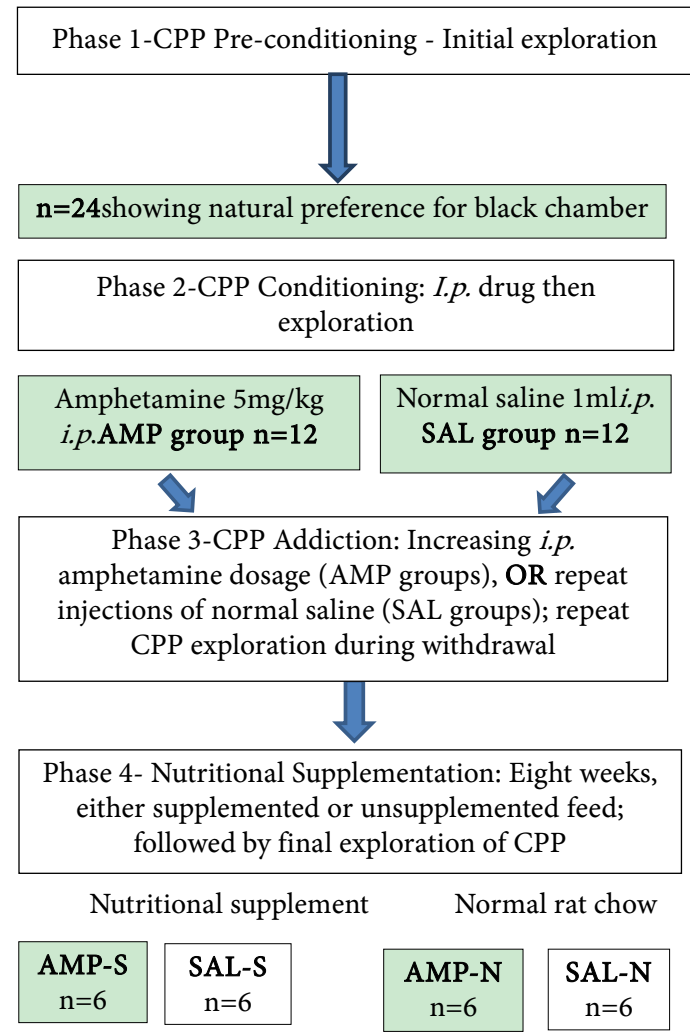

Figure 2. Flow diagram showing all phases of the experiment. 24 rats showing black-chamber preference were divided into two groups of 12 for treatment with amphetamine $5 \mathrm{mg} / \mathrm{kg}$ (AMP) or $0.9 \%$ normal saline (SAL). Six rats from each treatment group then were given nutritional supplementation.

- Group AMP-S: Amphetamine treated rats, fed with the nutritional supplement after the addiction phase

- Group AMP-N: Amphetamine treated rats, fed with standard rat chow throughout the experiment

- Group SAL-S: Saline treated rats, fed with the nutritional supplement after addiction phase

- Group SAL-N: Saline treated rats, fed with standard rat chow throughout the experiment

Rats in Groups AMP-S and SAL-S were individually housed in cages and received the nutritionally supplemented chow as first feed daily for the 8-week treatment period. Non-supplemented, standard rat chow was administered for the rest of the day, only after chow with supplement was fully consumed. The non-supplemented groups (i.e., Groups AMP-N and SAL-N) were administered food and water ad libitum throughout the day. Following this 8-week period, animals were randomly and individually placed in the CPP box and their performance in a final 20-minute exploration of the paradigm was assessed from video-recordings. Figure 2 is a graphical summary flow chart which explains the phases of the experiment. Data on time spent and entries in the drug-paired 
white chamber were collated for the different groups after aligning scores obtained to the numerical code assigned to the rats.

\section{Data Analysis}

Data on the mean percentage time (from a total time of 20 minutes) and entries (from the total entries into all arms of the CPP box) into the white CPP chamber by each animal were analyzed using the SPSS-19 software. Mean scores for white chamber percent entries and time were analyzed within groups using the $t$-test for pre-conditioning, the withdrawal period and after the 8 weeks of supplemented versus standard diet. Comparisons of the mean percentage time and entries into the white CPP chamber were also measured between groups, and analyzed using one-way ANOVA. Post-hoc analysis was performed using the Tukey test due to the distribution of the group means.

\section{Results}

\section{Pre-Conditioning}

A robust pre-conditioning milieu was created before drug treatment was initiated. Rats in all 4 treatment groups had significantly higher baseline percent entries into and time spent in the black chamber $(\mathrm{p}<0.05)$ prior to test conditions.

1) Within-group comparison of mean percent entries into the white CPP chamber for Groups AMP-S and AMP-N for all phases of the experiment

Amphetamine-treated rats in both AMP-S and AMP-N groups showed significantly increased percent entries into the drug-paired, white chamber of the CPP box during drug withdrawal, compared to their performance during pre-conditioning $(\mathrm{p}<0.001)$. This is demonstrated in Figure 3.

After 8 weeks of consuming the nutritional supplement, there was a significant decrease in percent entries into the drug-paired, white chamber $(p<0.05)$ by AMP-S rats, compared to SAL-S and SAL-N. There was also no significant difference between their initial pre-conditioning percent entries and those observed for the AMP-S rats after 8 weeks of nutritional supplementation.

On the other hand, non-supplemented, AMP-N rats continued to have significantly increased percent entries into the white chamber after 8 weeks of consuming standard rat chow $(\mathrm{p}<0.05)$.

2) Within-group comparison of mean percent time in the white CPP chamber for groups $A M P-S$ and $A M P-N$ for all phases of the experiment

All amphetamine-treated rats (AMP-S and AMP-N) showed significantly increased percent time in the drug-paired, white chamber of the CPP box during drug withdrawal, compared to their pre-conditioned time $(\mathrm{p}<0.001)$. This is demonstrated in Figure 4.

After 8 weeks of consuming the nutritional supplement, there was a significant decrease in percent time in the drug-paired chamber $(\mathrm{p}<0.05)$ by AMP-S rats. Also, there was no significant difference between the initial pre-conditioning white chamber percent time, and that noted after 8 weeks of supplementation. 


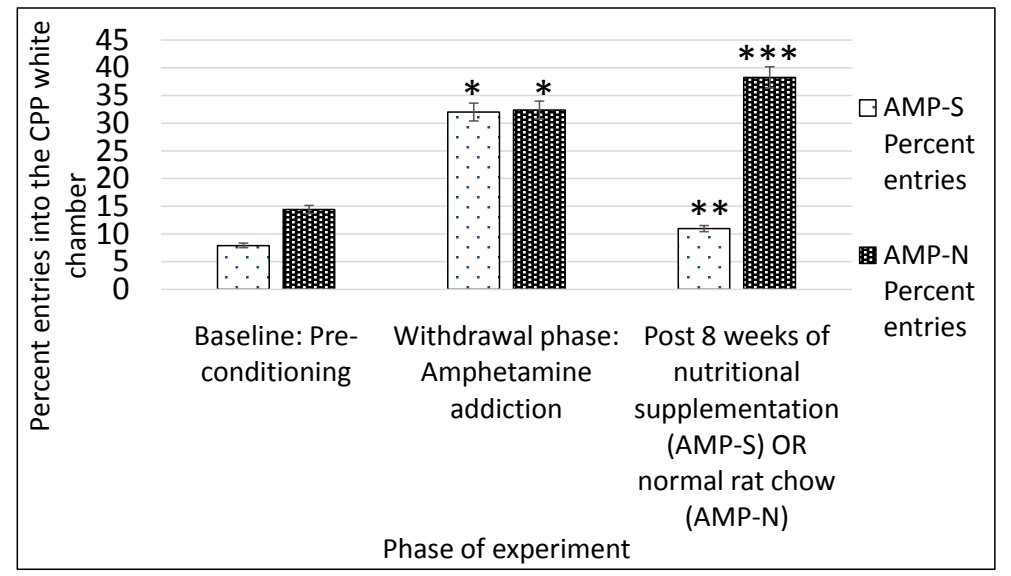

Figure 3. Graph of percent entries into the drug-paired white CPP chamber by amphetamine treated groups (AMP-S and AMP-N) for all 3 phases of the experiment: within-group comparisons. Comparison of percent entries into the white CPP chamber of rats in the amphetamine treated groups in all three phases of the experiment. ${ }^{*} \mathrm{p}<0.01$ Both AMP groups had significantly increased percent entries into the drug-paired white chamber during acute withdrawal compared to baseline exploration; ${ }^{* *} \mathrm{p}<0.01$ Group AMP-S rats exhibited a significant decrease in white chamber percent entries $(p<0.05)$ after nutritional supplementation, compared to their baseline percent entries. There was no significant difference between the white chamber percent entries of group AMP-S during pre-conditioning, and during post-supplementation. ${ }^{*} \mathrm{p}<0.01$ Group AMP-N continued significantly increased percent entries into the drug-paired white chamber after 8 weeks of normal rat chow.

On the other hand, rats in group AMP-N continued to show significantly increased percent time in the white chamber after 8 weeks of standard rat chow ( $\mathrm{p}$ $<0.001)$.

3) Comparison of mean percent entries into the white, drug-paired chamber for all treatment groups after drug treatment and after 8 weeks. between-group comparisons

After drug dosing and during the drug withdrawal period, amphetamine treated rats (Groups AMP-S and AMP-N) showed significantly higher percent entries into the drug-paired white chamber, compared to the saline treated groups, SAL-S and SAL-N $\left(\mathrm{F}_{(3,18)}=8.824 ; \mathrm{p}<0.001\right)$. This is shown in Figure 5.

However, after 8 weeks of nutritional supplementation, previously amphetamine addicted rats in Group AMP-S made significantly decreased percent entries into the previously drug-paired white chamber, compared to both saline treated groups, SAL-S and SAL-N $\left(\mathrm{F}_{(3,18)}=32.639 ; \mathrm{p}<0.001\right)$.

In contrast, following 8 weeks of standard, non-supplemented chow, amphetamine-addicted rats in Group AMP-N continued to show significantly increased percent entries into the previously drug paired white chamber $\left(\mathrm{F}_{(3,18)}=\right.$ 32.639; $\mathrm{p}<0.01)$ compared to saline treated groups, SAL-S and SAL-N.

4) Between-group comparison of mean percent time in the white, drug-paired chamber for all treatment groups during withdrawal and after 8 weeks 


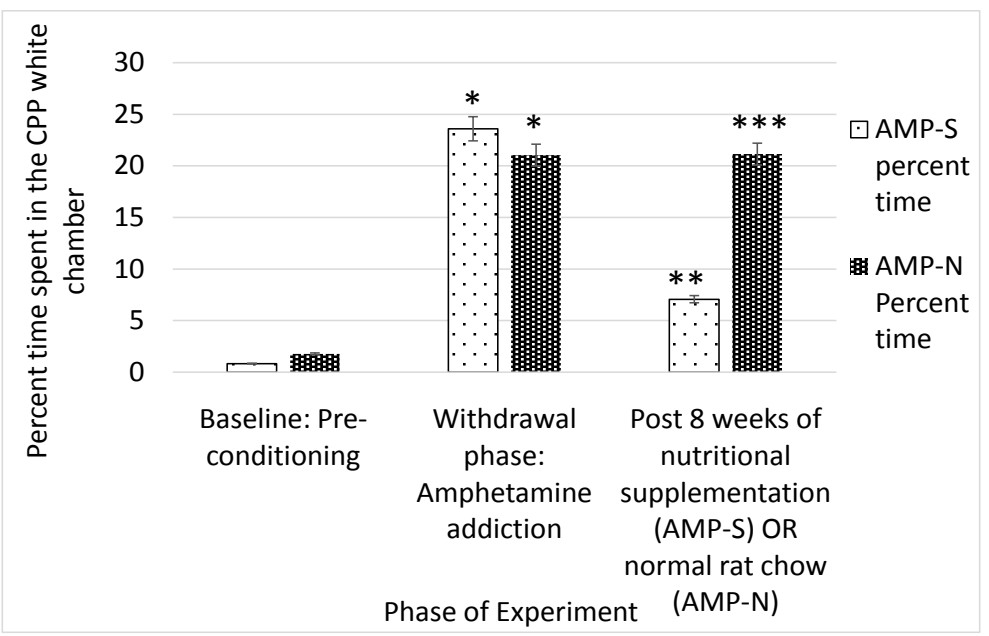

Figure 4. Graph of percent time spent in the drug-paired white CPP chamber by amphetamine treated groups (AMP-S and AMP-N) for all 3 phases of the experiment: within-group comparisons. Comparison of percent time spent in the white CPP chamber by the amphetamine treated groups in all three phases of the experiment. ${ }^{*} \mathrm{p}<0.001$ Both AMP groups had significant increases in percent time in the drug-paired white chamber during acute drug withdrawal, compared to baseline. ${ }^{* *} \mathrm{p}<0.001$ Group AMP-S exhibited a significant decrease in white chamber percent time $(\mathrm{p}<0.05)$ after nutritional supplementation, compared to during withdrawal. ${ }^{\star * *} \mathrm{p}<0.01$ Group AMP-N continued to spend significantly higher percent time in the drug-paired white chamber after 8 weeks of standard rat chow, compared to during withdrawal. There was no significant difference between the time spent in the white chamber by group AMP-S during pre-conditioning, and during post-supplementation.

During drug withdrawal, amphetamine treated rats (Groups AMP-S and AMP-N) spent a significantly increased percent time in the drug-paired white chamber, compared to the saline treated groups (SAL-S and SAL-N; $\mathrm{F}_{(3,18)}=$ 7.025; $\mathrm{p}<0.001)$. This is shown in Figure 6.

After 8 weeks of nutritional supplementation, previously amphetamine addicted rats in Group AMP-S spent significantly decreased percent time in the previously drug-paired white chamber, compared to both supplemented and non-supplemented, saline treated groups (SAL-S and SAL-N; $\mathrm{F}_{(3,18)}=10.251 ; \mathrm{p}=$ 0.006).

After 8 weeks of standard, non-supplemented chow, amphetamine-addicted rats in Group AMP-N continued to have a significantly higher percent time in the drug paired white chamber $\left(\mathrm{F}_{(3,18)}=10.251 ; \mathrm{p}=0.006\right)$ compared to saline treated groups, i.e. SAL-S and SAL-N.

\section{Discussion}

\subsection{Summary of Main Findings}

The results of this study show for the first time, that complete reversal of amphetamine drug-seeking behavior in stimulant addicted animals can be demonstrated solely after application of a nutritional intervention. 


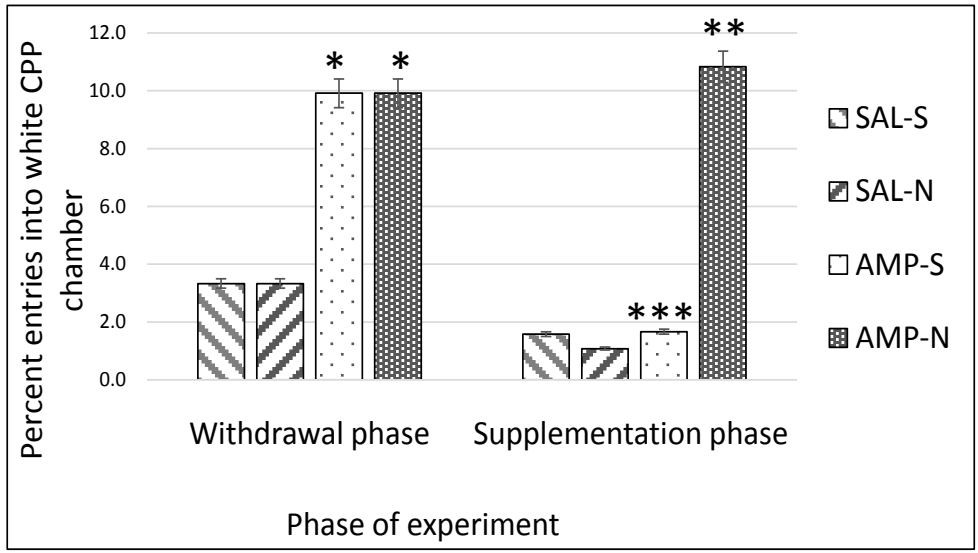

Figure 5. A comparison of percent entries into the drug-paired white CPP chamber by amphetamine treated (AMP) and saline treated (SAL) rat groups during withdrawal and following 8 weeks of nutritional supplementation (AMP-S and SAL-S) or standard rat chow (AMP-N and SAL-N). Comparison of the percent entries into the white CPP chamber for all groups during the withdrawal phase and after 8 weeks of either nutritional supplementation (AMP-S and SAL-S) or normal rat chow (Groups AMP-N and SAL-N). ${ }^{*} \mathrm{p}<$ 0.01 AMP groups made significantly higher percent entries into the drug-paired white chamber then vehicle (SAL) groups during withdrawal. ${ }^{* *} \mathrm{p}<0.01$ Group AMP-N continued to make significantly higher percent entries into the white chamber than saline (SAL) groups after 8 weeks of standard, un-supplemented rat chow. ${ }^{* *} \mathrm{p}<0.01$ Group AMP-S made significantly lower percent entries into the white, drug-paired chamber than AMP-N after 8 weeks of nutritional supplementation.

The widely accepted criteria for acquisition of addiction using the conditioned place preference paradigm were evident in our baseline results. At baseline, all of the animals spent significantly less time in the white chamber, than they did in the black, naturally preferred chamber. Amphetamine administration paired with the white, non-preferred CPP chamber significantly increased the percent entries into, and percent time spent in the otherwise aversive white chamber during withdrawal $(p<0.05)$. Eight weeks of rat chow re-constituted to incorporate the formulated nutritional supplement resulted in the behavioral transition to significantly fewer entries into, and time spent in the drug-paired white chamber $(\mathrm{p}<0.05)$. Amphetamine addicted, nutritionally supplemented animals performed similarly to the saline-treated, drug-naïve animals in white chamber time and entries. The saline treated controls maintained "baseline" white chamber aversion throughout the experiment.

The results of this study have the potential to profoundly revolutionize therapeutic management of drug addiction. The multi-nutrient supplement regimen, which previously anecdotally diminished withdrawal symptoms in human alcoholics and heroin addicts, has been shown in this study to eliminate drug-seeking behavior by amphetamine addicted rodents in the CPP paradigm, a valid, reliable and frequently used animal model of drug addiction. 


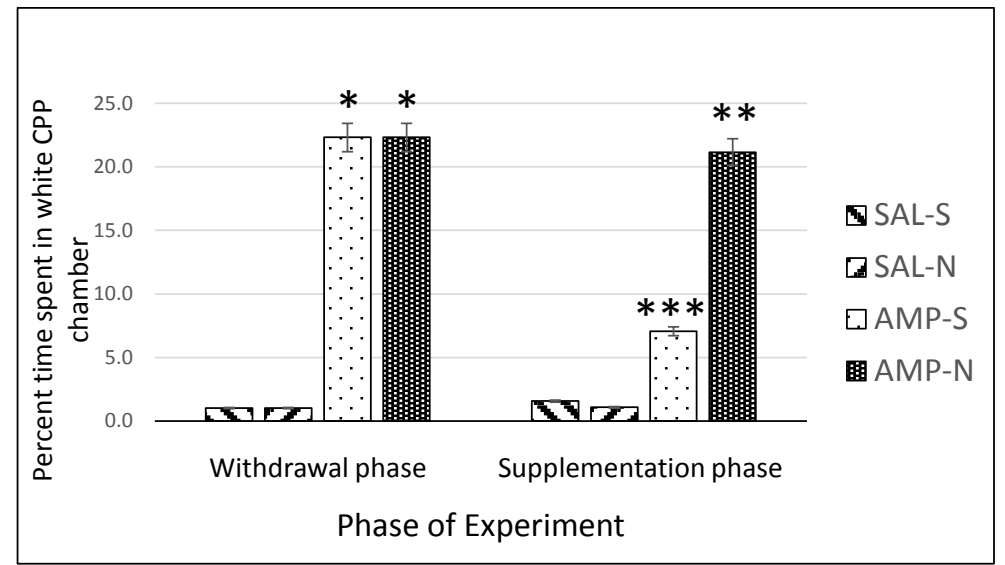

Figure 6. Comparison of percent time spent in the drug-paired white CPP chamber by amphetamine treated (AMP) and saline treated (SAL) groups during withdrawal and then after 8 weeks of nutritional supplementation or un-supplemented standard rat chow. Comparison of the percent time in the white CPP chamber for all groups during the withdrawal phase and after 8 weeks of either nutritional supplementation (AMP-S and SAL-S) or normal rat chow (Groups AMP-N and SAL-N). ${ }^{*} \mathrm{p}<0.01$ Groups AMP had significantly higher percent time in the drug paired white chamber than control (SAL) groups. ${ }^{* *} \mathrm{p}<0.01$ Group AMP-N had significantly higher percent time in the drug-paired white chamber after eight weeks of standard, un-supplemented rat chow. ${ }^{* * *} \mathrm{p}<0.01$ Group AMP-Sspent significantly less percent time in the drug paired white chamber after eight weeks of nutritional supplementation than did the un-supplemented, previously addicted group AMP-N.

\subsection{Role of Nutrition in Addiction Recovery}

These results complement those of an extensive, 50-year review of the literature related to nutrition as therapy in drug abuse recovery done previously [12]. They corroborate evidence that vitamin, mineral and amino acid therapy can reduce withdrawal symptoms, increase treatment retention and quality of life and contribute to higher abstinence rates in stimulant addicted subjects [12]. The rehabilitative role of amino acid supplementation with tyrosine and tryptophan, aimed at restoration of catecholamine balance in creating a foundation for complete recovery, has also been previously discussed [13].

A cutting-edge review of the neurobiology of addiction recognizes three stages of the disease, and affirms that each stage influences and is fed by disordered circuitry in different brain regions. In the binge/intoxication stage, previously neutral stimuli become associated with drug availability, promoting habit formation that fosters excessive drug seeking via increases in dopamine and glutamate neurotransmission. Those authors associate this first stage with diminished reward function via dopamine and opioid peptide deficits (2). The animals in this study were likely confined to that initial stage of the continuum of drug addiction, as we employed a relatively short time line. The human chronic drug addict, however, would also likely have derangements in executive function via the dysregulation of glutamatergic, GABAergic, and dopaminergic neuronal 
networks in the prefrontal cortex (2).

Bearing in mind the gut and neuronal derangements associated with stimulant addiction, it is likely that the balance of fatty acids, minerals and vitamins in this supplement may potentially replenish catecholamine neurotransmitters [14]. It may also contribute to the restoration of gut health and neuronal cellular function in order to improve CNS regulatory mechanisms and restore normal decisions to behave as if there had been no drug exposure.

A nutritional supplement that contained fish oil (5.6 grams a day); a multiple vitamin; a brain supplement with ginkgo and vinpocetine; acetylcholine (acetyl1-carnitine and huperzine A), and antioxidant (alpha-lipoic acid and n-acetylcysteine) was found to play a significant role in substance abuse rehabilitation and brain injury reversal [15]. These authors reported on improvements in cognitive functions of attention, memory, reasoning and speed of information processing. Brain SPECT scans provided information on brain perfusion showing improvements in cerebral blood flow also. Our results complement those findings, but we have demonstrated more effectively the ability of our supplement to significantly decrease drug seeking behaviour.

\subsection{Strengths and Limitations}

Our work demonstrated unequivocally that rats became amphetamine addicts, as we employed the international gold standard for addiction studies. Our method of including the nutritional supplement in the first feed of each day enabled the results to be more uniform, regardless of how much each animal consumed. The limitations are that we can only interpret cue-directed animal behavior in terms of positive CPP or drug-seeking behavior; and negative CPP or drug aversion. The physiological derangements and other qualitative and emotional aspects of human addiction may not have been modeled in our study.

This study opens the door to continued behavioral analysis of nutrient therapy for drug addiction. Much scope exists for inclusion of this supplement in stimulant drug abuse rehabilitation programs.

\section{Acknowledgements}

The team wishes to thank the Mona Campus Office of Graduate Studies and Research at UWI for providing funding for the study. Many thanks also to Dr. Salako-Akande for making available her proprietary formulation of the nutritional supplement that was used in this research.

\section{References}

[1] American Psychiatric Association (2013) Diagnostic and Statistical Manual of Mental Disorders. 5th Edition, American Psychiatric Publishing, Washington DC.

[2] Koob, G.F. and Volkow, N.D. (2016) Neurobiology of Addiction: A Neurocircuitry Analysis. The Lancet Psychiatry, 3, 760-773. https://doi.org/10.1016/S2215-0366(16)00104-8

[3] Volkow, N.D. and Baler, R.D. (2014) Addiction Science: Uncovering Neurobiologi- 
cal Complexity. Neuropharmacology, 76, 235-249.

https://doi.org/10.1016/j.neuropharm.2013.05.007

[4] Gilon, P. (2016) Cocaine- and Amphetamine-Regulated Transcript: A Novel Regulator of Energy Homeostasis Expressed in a Subpopulation of Pancreatic Islet Cells. Diabetologia, 59, 1855-1859. https://doi.org/10.1007/s00125-016-4052-y

[5] Lau, J. and Herzog, H. (2014) CART in the Regulation of Appetite and Energy Homeostasis. Frontiers in Neuroscience, 8, 313. https://doi.org/10.3389/fnins.2014.00313

[6] Mehler, P.S. and Brown, C. (2015) Anorexia Nervosa-Medical Complications. Journal of Eating Disorders, 3, 11. https://doi.org/10.1186/s40337-015-0040-8

[7] Parrish, C.R. and Yoshida, C.M. (2005) Nutrition Intervention for the Patient with Gastroparesis: An Update. Practical Gastroenterology, 29, 29.

[8] Wiss, D. and Waterhouse, S. (2014) Nutrition Therapy for Eating Disorders, Substance Use Disorders, and Addictions. Eating Disorders, Addictions and Substance Use Disorders, 509-532. https://doi.org/10.1007/978-3-642-45378-6_23

[9] Tzschentke, T.M. (1998) Measuring Reward with the Conditioned Place Preference Paradigm: A Comprehensive Review of Drug Effects, Recent Progress and New Issues. Progress in Neurobiology, 56, 613-672.

https://doi.org/10.1016/S0301-0082(98)00060-4

[10] Papp, M., Gruca, P. and Willner, P. (2002) Selective Blockade of Drug-Induced Place Preference Conditioning by ACPC, a Functional NDMA-Receptor Antagonist. Neuropsychopharmacology, 27, 727-743. https://doi.org/10.1016/S0893-133X(02)00349-4

[11] Gardner, N.S., Luke, K.S.J., Wheatley, A.O., De La Haye, W., Bahado-Singh, P., Dilworth, D., McGrowder, D.A., Barton, E., Young, L.E., Salako-Akande, A., Morrison, E., Eldemire-Shearer, D., Lowe, H. and Asemota, H. (2015) Plasma Cocaine Metabolite and Liver CYP4503A4 Isoenzyme Levels as Indicators of Cocaine Dependence in Rats Treated with Nutritional Supplements. International Journal of Measurement Technologies and Instrumentation Engineering, 5, 29-43. https://doi.org/10.4018/IJMTIE.2015070103

[12] Marquez, P., Nguyen, A.T., Hamid, A. and Lutfy, K. (2008) The Endogenous OFQ/N/ORL-1 Receptor System Regulates the Rewarding Effects of Acute Cocaine. Neuropharmacology, 54, 564-568.

[13] Kerr, K. and Cecchini, M. (1991) Are We Ignoring Effective Substance Abuse Treatment Solutions? Vitamin Therapy as an Essential Treatment Component. Presentation Abstract, 3rd International Conference on Chemical Contamination and Human Detoxification, Hunter College, New York.

[14] Grotzkyj-Giorgi, M. (2009) Nutrition and Addiction-Can Dietary Changes Assist with Recovery? Drugs and Alcohol Today, 9, 24-28. https://doi.org/10.1108/17459265200900016

[15] Amen, D.G., Wu, J.C., Taylor, D. and Willeumier, K. (2011) Reversing Brain Damage in Former NFL Players: Implications for Traumatic Brain Injury and Substance Abuse Rehabilitation. Journal of Psychoactive Drugs, 43, 1-5.

https://doi.org/10.1080/02791072.2011.566489 